\title{
Article \\ Ultrahigh Spin Filter Efficiency, Giant Magnetoresistance and Large Spin Seebeck Coefficient in Monolayer and Bilayer Co-/Fe-/Cu-Phthalocyanine Molecular Devices
}

\author{
Jianhua Liu ${ }^{1,2} \mathbb{D}$, Kun Luo ${ }^{1,2}$, Hudong Chang ${ }^{1,2}$, Bing Sun ${ }^{1,2, *}$ and Zhenhua Wu ${ }^{1,2, *(D)}$ \\ 1 Institute of Microelectronics of Chinese Academy of Sciences, Beijing 100029, China; \\ liujianhua9@ime.ac.cn (J.L.); luokun@ime.ac.cn (K.L.); changhudong@ime.ac.cn (H.C.) \\ 2 College of Microelectronics, University of Chinese Academy of Sciences, Beijing 100029, China \\ * Correspondence: sunbing@ime.ac.cn (B.S.); wuzhenhua@ime.ac.cn (Z.W.); Tel.: +86-010-82995593 (B.S.); \\ +86-010-82995791 (Z.W.)
}

check for

updates

Citation: Liu, J.; Luo, K.; Chang, H.; Sun, B.; Wu, Z. Ultrahigh Spin Filter Efficiency, Giant Magnetoresistance and Large Spin Seebeck Coefficient in Monolayer and Bilayer $\mathrm{Co}-/ \mathrm{Fe}-/ \mathrm{Cu}-\mathrm{Ph}$ thalocyanine Molecular Devices. Nanomaterials 2021, 11, 2713. https://doi.org/ 10.3390/nano11102713

Academic Editors: Maurizio Casarin and Mads Brandbyge

Received: 29 August 2021

Accepted: 11 October 2021

Published: 14 October 2021

Publisher's Note: MDPI stays neutral with regard to jurisdictional claims in published maps and institutional affiliations.

Copyright: (c) 2021 by the authors. Licensee MDPI, Basel, Switzerland. This article is an open access article distributed under the terms and conditions of the Creative Commons Attribution (CC BY) license (https:/ / creativecommons.org/licenses/by/ $4.0 /)$.

\begin{abstract}
The spin related electrical and thermoelectric properties of monolayer and bilayer MPc $(\mathrm{M}=\mathrm{Co}, \mathrm{Fe}, \mathrm{Cu})$ molecular devices in a parallel spin configuration (PC) and an anti-parallel spin configuration (APC) between the V-shaped zigzag-edged graphene nanoribbon electrodes and the center bilayer MPc molecules are investigated by combining the density functional theory and nonequilibrium Green's function approaches. The results show that there is an ultrahigh spin filter efficiency exceeding $99.99995 \%$ and an ultra-large total conductance of $0.49996 \mathrm{G}_{0}$ for FePc-CoPc molecular devices in the PC and a nearly pure charge current at high temperature in the APC and a giant MR ratio exceeding $9.87 \times 10^{6 \%}$ at a zero bias. In addition, there are pure spin currents for $\mathrm{CuPc}$ and FePc molecular devices in the PC, and an almost pure spin current for FePc molecular devices in the APC at some temperature. Meanwhile, there is a high SFE of about $99.99585 \%$ in the PC and a reserved SFE of about $-19.533 \%$ in the APC and a maximum MR ratio of about $3.69 \times 10^{8 \%}$ for the FePc molecular device. Our results predict that the monolayer and bilayer MPc $\left(\mathrm{M}=\mathrm{Co}_{\mathrm{r}}\right.$ $\mathrm{Fe}, \mathrm{Cu}$ ) molecular devices possess large advantages in designing high-performance electrical and spintronic molecular devices.
\end{abstract}

Keywords: bilayer metal phthalocyanine; molecular device; spin filter efficiency; giant magnetoresistance; spin Seebeck coefficient

\section{Introduction}

Molecular spintronic devices have been extensively studied in the past decade, and are a hopeful approach to downsize spintronic devices and are expected to be widely used in high-density data storage and quantum computing [1,2]. A large spin-filter efficiency (SFE) could be generated in molecular spintronic devices [3-5]. For example, a significant spin-filtering effect was found in Mn-Pc and Fe-Pc with single-walled carbon nanotube (SWCNT) electrodes [6]; the SFE of the chromium-phthalocyanine molecular device with zigzag graphene nanoribbon (ZGNR) electrodes is nearly $100 \%$ in a wide bias voltage region [7]. For a bilayer $\mathrm{CuPc}$ molecular device, changing the twist angle between the two molecules could also obtain a high SFE [8]. The spin-dependent hybridization of the electrode and molecular orbitals could cause a large magnetoresistance [9]. A molecular junction made of two MnPc molecules linked by single-walled carbon nanotubes shows perfect spin filter effects and an giant magnetoresistance (GMR) [10]. A cyclooligomeric Mn-phthalocyanine dimer molecular junction shows high-efficiency dual spin-filtering [11]. The interaction of spins with heat currents was studied in MPc (M = Mn, Fe, Co, Ni) molecular devices and the MnPc device exhibits a perfect SFE and thermal-SFE as well as sizeable GMR/thermal GMR effects [12]. The metal phthalocyanine has also been used in photodetectors and nano-porous $\mathrm{GaN}$ and $\mathrm{CoPc} p-n$ vertical heterojunction bonded as a high-performance self-powered ultraviolet photodetector [13]. 
In this paper, we studied the spin-related electrical and thermoelectric properties of monolayer and bilayer MPc $(\mathrm{M}=\mathrm{Fe}, \mathrm{Co}, \mathrm{Cu})$ molecular device in the PC and APC with two V-shaped zigzag-edged graphene nanoribbons (ZGNRs) electrodes by employing LDA, LDA + U and the non-equilibrium Green's function (NEGF) in combination with the density functional theory (DFT) $[14,15]$. These methods have their advantages over other approaches for nanomaterials with transition metals (TM), Fe in particular. The optimized bond lengths between the $3 \mathrm{~d}$ metal center and the nearest nitrogen atoms for Fe-/Co-/Cu-phthalocyanines in the gas phase in our NanoDCAL programs [16], LDA and $\mathrm{LDA}+\mathrm{U}$, are close to the experimental values [17-19]. Comparing these with traditional metal electrode, SWCNTs employed as the electrodes for molecular devices could create covalent bridging between electrodes and conducting molecules and have a better performance [20-23]. The SWCNTs were chosen as electrodes of a series of $3 \mathrm{~d}$ transition metal(II) phthalocyanines (MPc, M = Mn, Fe, Co, Ni, Cu and Zn) in Reference [6] and only MnPc and FePc could act as nearly perfect spin filters. Due to their quasi-one-dimensional structure and their excellent electronic transport properties, GNRs have also been a potential candidate for conductive electrodes [23-25]. The spin-transport properties of molecular devices constructed using hydrogen-phthalocyanine and transition metal (TM)-phthalocyanine molecules with zigzag graphene nanoribbon (ZGNR) electrodes are investigated in Reference [7] and the results show that there exists a giant magnetoresistance in both the hydrogen-phthalocyanine and TM-phthalocyanine systems. Comparing with the SWCNT electrodes in Reference [6] and the ZGNR electrodes in Reference [7], the V-shaped ZGNR electrodes allow the SFE and MR of our monolayer Co-/Fe-/Cu-phthalocyanine molecular devices under equilibrium states to exceed $99.998 \% / 99.9958 \% / 97 \%$ in the PC and $2.59 \times 10^{7 \%} / 2.58 \times 10^{7} \% / 3.069 \times 10^{8} \%$, respectively. The SFE of the bilayer CoPc and FePc devices exceed $99.9998 \%$ and the SFE of the bilayer CuPc molecular device is over 99.99996\%, which exceeds those of the bilayer FePc, bilayer CoPc and FePc-CoPc molecular devices. In addition, the SFE of the CuPc-FePc and CuPc-CoPc devices exceed 99.999997\%, which are perfect SFEs. In addition, the spin-down and total conductance and SFE of our bilayer Co-/Fe-/Cu-phthalocyanine molecular devices in the PC are bigger than the ones in our monolayer $\mathrm{Co}-/ \mathrm{Fe}-/ \mathrm{Cu}$-phthalocyanine molecular devices, the spin-down channel dominates the transmission and density of state at Fermi energy in the PC. There are large pure spin currents in the monolayer $\mathrm{CuPc}$ and FePc molecular devices in the PC at some temperatures. Additionally, the spin-dependent charge Seebeck coefficient and the spin Seebeck coefficient are almost equal for $\mathrm{CoPc}, \mathrm{CuPc}-\mathrm{CuPc}, \mathrm{CoPc}-\mathrm{CoPc}, \mathrm{FePc}-\mathrm{FePc}, \mathrm{CuPc}-\mathrm{CoPc}$, $\mathrm{CuPc}-\mathrm{FePc}$, and CoPc-FePc molecular devices in the PC due to the spin-down Seebeck coefficient being close to zero and there are usually large charge Seebeck coefficients at high temperatures for the abovementioned molecular devices in the APC. Physical mechanisms are proposed for these phenomena. The calculated transmission spectra and the real-space scattering states of the modeled mono- and bilayers can be used for future experimental photoemission spectroscopy and other studies. These theoretical calculations predict new monolayer and bilayer phthalocyanine-based molecular devices with high MR and Seebeck coefficients. Based on the calculated spin-dependent conductance and other characteristics, ultrahigh SFE, GMR, and Seebeck coefficients for Co-/Fe-/Cu-phthalocyanine molecular devices are predicted for the first time. This makes the results motivating for further experimental studies of phthalocyanine- and TM-layered structures. The obtained large SFE of the parallel spin configuration can be utilized in various molecular spintronic devices.

\section{Methods}

We investigated the spin transport properties of monolayer and bilayer $\mathrm{MPc}(\mathrm{M}=\mathrm{Fe}$, $\mathrm{Co}, \mathrm{Cu}$ ) molecular devices by combining the density functional theory and non-equilibrium Green's function approach, as implemented by the NanoDCAL transport package [16,26]. Figure $1 \mathrm{a}$ and $\mathrm{b}$ show top and side views of the structure of themonolayer and bilayer MPc $(\mathrm{M}=\mathrm{Cu}, \mathrm{Fe}, \mathrm{Co})$ molecular devices. The device was formed of three parts: left and right electrodes (which extended to $\pm \infty$ ) and the central scattering region, which contained MPc 
molecules, in the left and right buffer layers. The energy cutoff was set to 150 Rydberg, the k-point grid was set to $100 \times 1 \times 1$ and electrode temperature was chosen to be $300 \mathrm{~K}$. The electrodes of ZGNRS were modeled with a supercell $(4.9332 \AA \times 32.821 \AA \times 18 \AA$ for the monolayer MPc and $4.9332 \AA \times 32.821 \AA \times 18 \AA$ for the bilayer MPc, the distance between the bilayer MPc was $3 \AA$ ) subjected to periodic boundary conditions. A vacuum layer about $15 \AA$ in the y direction and $\mathrm{z}$ direction was introduced to eliminate interactions between GNRs in neighboring cells and the edge atoms; both electrodes and central region were saturated with hydrogen $(\mathrm{H})$ atoms to remove the dangling bonds [23]. The exchangecorrelation function is described by the local density approximation (LDA) proposed by Perdew and Zunger, and a plus U correction [27] (LDA + U) was used for the above calculations, considering the localized 3d-orbital of Fe $(\mathrm{U}=3 \mathrm{eV})$ and $\mathrm{Co}(\mathrm{U}=2 \mathrm{eV})$ atoms [28]. Quantum transport phenomena with the monolayer and bilayer MPc molecular devices were further understood by analyzing the transmission spectrum, projected density of states, and the scattering states.

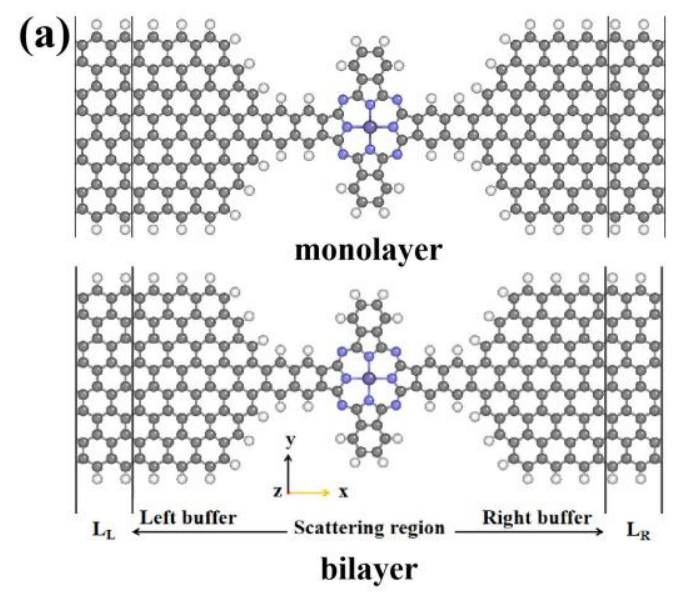

(b)

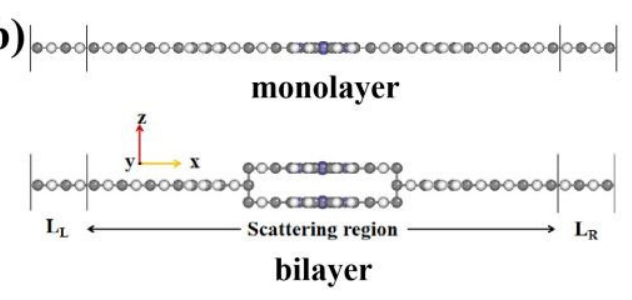

Figure 1. The structure of the monolayer and bilayer $\mathrm{MPc}(\mathrm{M}=\mathrm{Cu}, \mathrm{Fe}, \mathrm{Co})$ molecular devices. The navy, blue, black, and grey balls represent $\mathrm{M}, \mathrm{N}, \mathrm{C}$, and $\mathrm{H}$ atoms, respectively. The molecule in the scattering region is MPc, the electrode is V-shaped zigzag-edged GNRs. $(\mathbf{a}, \mathbf{b})$ are the top and side view of the molecular device.

Spin-polarized zero-bias conductance is given by the Landauer-Buttiker formula [29]

$$
G_{\sigma}=\frac{e^{2}}{h} T_{\sigma}\left(E_{F}\right)
$$

where $T_{\sigma}\left(E_{F}\right)$ is the electron transmission coefficient for the spin-up ( $\uparrow$ ) and spin-down $(\downarrow)$ electrons $(\sigma=\uparrow / \downarrow)$ and $E_{F}$ is the Fermi level.

At zero bias, SFE is defined as:

$$
S F E=\frac{T_{\downarrow}\left(E_{F}\right)-T_{\uparrow}\left(E_{F}\right)}{T_{\uparrow}\left(E_{F}\right)+T_{\downarrow}\left(E_{F}\right)} \times 100 \%
$$

where $T_{\uparrow}\left(E_{F}\right)$ and $T_{\downarrow}\left(E_{F}\right)$ stand for the transmission coefficient of the spin-up (SU) and spin-down (SD) states at the Fermi level, respectively.

Considering the spin direction of the lead, there is a giant magnetoresistance (MR) effect and the MR ratio for the device for the PC and APC are defined as: 


$$
M R=\frac{T_{P C}-T_{A P C}}{T_{A P C}} \times 100 \%
$$

where $T_{P C}=T_{P C}\left(E_{F}\right)_{\uparrow}+T_{P C}\left(E_{F}\right)_{\downarrow}, T_{A P C}=T_{A P C}\left(E_{F}\right)_{\uparrow}+T_{A P C}\left(E_{F}\right)_{\downarrow}$.

Considering the spin-dependent thermoelectric properties of the monolayer and bilayer MPc $(\mathrm{M}=\mathrm{Fe}, \mathrm{Co}, \mathrm{Cu})$ molecular devices, the usual charge Seebeck coefficient $\left(\mathrm{S}_{\mathrm{C}}\right)$ and the spin Seebeck coefficient $\left(S_{S}\right)$ are defined as $S_{C}=\left(S_{\uparrow}+S_{\downarrow}\right) / 2$ and $S_{S}=\left(S_{\uparrow}-S_{\downarrow}\right) / 2$, where $S_{\uparrow}$ and $S_{\downarrow}$ are the SU and SD Seebeck coefficients, respectively [30].

\section{Results and Discussion}

By comparing the optimized bond lengths between the $3 \mathrm{~d}$ metal center $(\mathrm{M})$ and the nearest nitrogen $(\mathrm{N})$ atoms for $\mathrm{Fe}-/ \mathrm{Co}-/ \mathrm{Cu}$-phthalocyanines in the gas phase in our NanoDCAL program LDA (for CuPc) and LDA + U (for FePc and CoPc) results with calculations for the same systems done with the SIESTA + SMEAOGOL programs and the PBE and PBEh functionals in Reference [6] as well as the experimental values in Table 1. We found that our results were close to the experimental values and our NanoDCAL program $\mathrm{LDA}(+\mathrm{U})$ was very characteristic for Fe-/Co-/Cu-phthalocyanines molecular devices.

Table 1. Optimized bond lengths between the $3 \mathrm{~d}$ metal center $(\mathrm{M})$ and the nearest nitrogen $(\mathrm{N})$ atoms for Fe-/-Co-/-Cu-phthalocyanines in the gas phase. The experimental values are also given for comparison.

\begin{tabular}{cccccc}
\hline \multicolumn{6}{c}{ M-N (Å) } \\
\hline \multicolumn{7}{c}{ Gaussian 03 } \\
\hline \multirow{2}{*}{ MPc molecule } & SIESTA & PBE & PBEh & LDA(+U) & Exp. \\
FePc & Ref. [6] & Ref. [6] & Ref. [6] & Our work & 1.927 (Ref. [17]) \\
CoPc & 1.943 & 1.934 & 1.941 & $1.937(+\mathrm{U})$ & 1.908 (Ref. [18]) \\
CuPc & 1.931 & 1.934 & 1.932 & $1.893(+\mathrm{U})$ & 1.932 (Ref. [19]) \\
\hline
\end{tabular}

For monolayer CoPc, FePc, CuPc, bilayer CuPc-CuPc, FePc-FePc, CoPc-CoPc, FePc$\mathrm{CoPc}, \mathrm{CoPc}-\mathrm{CuPc}$ and $\mathrm{CuPc}-\mathrm{FePc}$ molecular devices (MDs) at zero bias, Figure 2a shows that the spin-up (SU) conductance of the MPc for the PC is notably smaller than the spindown (SD) one. The SD G and the total G of the FePc-CoPc MD and SU G of CuPc MD in the PC, total G of CuPc-FePc MD, SU G of CuPc-CoPc MD and SD G of CuPc-CuPc $\mathrm{MD}$ in the APC are maximum for the above MPc(s) MDs, respectively. The SD G and total $\mathrm{G}$ of bilayer $\mathrm{MPc}(\mathrm{s}) \mathrm{MD}$ are larger than the ones of the monolayer MPc MD in the PC and APC, respectively. Based on Equation (1), the change rule of the transmission at the Fermi level of the MPc(s) MD is perfectly consistent with that of the conductance of the $\operatorname{MPc}(\mathrm{s})$ shown in Figure 2b. The physical mechanism of the conductance change law can be understood by analyzing the transmission spectra, scattering states, and projected density of state as follows:

For CoPc, CuPc, FePc, CuPc-CuPc, FePc-FePc, CoPc-CoPc, FePc-CoPc, CuPc-CoPc, and $\mathrm{FePc}-\mathrm{CuPc}$ molecular devices at zero bias, the SFE in the $\mathrm{PC}$ are all close to $100 \%$ and there is a maximum SFE of $99.999998 \%$ for CuPc-CoPc MD in Figure 3. In the APC, the SFE of all MPc MDs are relatively small and there are negative SFEs for $\mathrm{CuPc}, \mathrm{FePc}, \mathrm{FePc}-\mathrm{FePc}$, $\mathrm{CoPc}-\mathrm{CoPc}$, CuPc-CoPc and FePc-CuPc MDs, which means that their SU transmission is greater than $\mathrm{SD}$ transmission and the spin polarization is reversed. There is a minimum SFE of about $-24.183 \%$ for FePc-FePc MD. The SFE is determined by the transmission in the SU and SD channel at the Fermi level based on Equation (2). Figure 3 also shows that there is a giant MR effect in MPc(s) MD. There is a maximum MR ratio of about $3.69 \times 10^{8 \%}$ for the monolayer FePc MD, and the MR ratio of FePc-FePc MD is largest in the bilayer MPcs MD. 


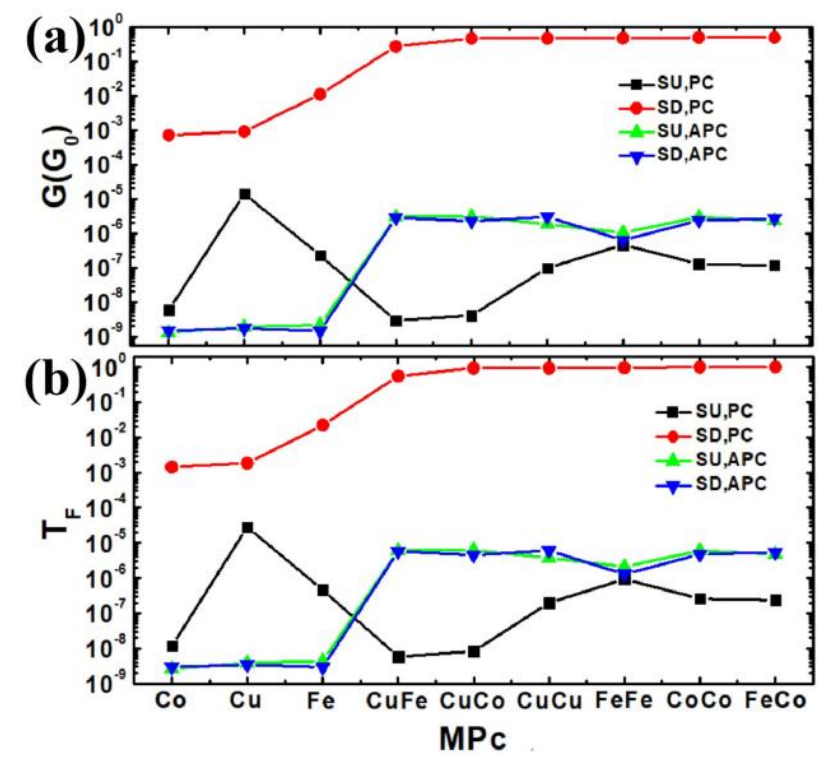

Figure 2. The conductance $G(\mathbf{a})$ and transmission at the Fermi level (b) of the MPc molecular device for monolayer $\mathrm{CoPc}, \mathrm{CuPc}$, FePc, bilayer $\mathrm{CuPc}-\mathrm{FePc}, \mathrm{CuPc}-\mathrm{CoPc}, \mathrm{CuPc}-\mathrm{CuPc}$, FePc-FePc, CoPc-CoPc, and FePc-CoPc at zero bias.

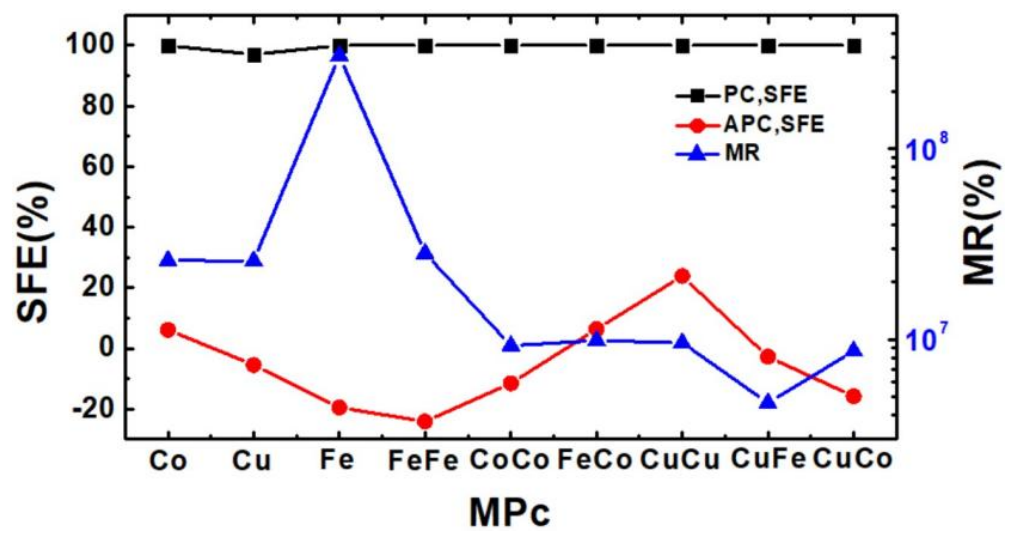

Figure 3. The SFE and MR of the MPc molecular device for the monolayer $\mathrm{CoPc}, \mathrm{CuPc}, \mathrm{FePc}$, bilayer $\mathrm{CuPc}-\mathrm{FePc}, \mathrm{CuPc}-\mathrm{CoPc}, \mathrm{CuPc}-\mathrm{CuPc}, \mathrm{FePc}-\mathrm{FePc}, \mathrm{CoPc}-\mathrm{CoPc}$, and FePc-CoPc at zero bias.

To better understand the transport properties of the MPc molecular devices, we investigated transmission and the projected density of state (projected onto orbitals with considering the angular momentum quantum number, that is AMQN) in the SU and SD channels among FePc and FePc-FePc molecular devices in the PC and APC under equilibrium states, as shown in Figure 4 (there are two main SU and three main transmission peaks from $-1 \mathrm{eV}$ to $1 \mathrm{eV}$ at zero bias: $-0.82 \mathrm{eV}, 0.08 \mathrm{eV}, 0.99 \mathrm{eV}$ for SD and the SU transmission peak is $-0.82 \mathrm{eV}, 0.98 \mathrm{eV}$ ). Owing that the origin of the transmission peaks could be figured out by projected density of states (PDOS) [31], as shown in Figure 4a, there were several obvious PDOS peaks of $\mathrm{p}$ - and d-orbitals, some of them corresponded basically to the transmission peaks at or around the same energy. For the FePc MDs in the APC shown in Figure 4c, the corresponding situation of the PDOS peaks and transmission peaks is similar to the one in Figure $4 \mathrm{a}$. However, Figure $4 \mathrm{~b}$ shows that there are many PDOS peaks of s-, p- and d-orbitals, and there are only three obvious SU transmission peaks and two obvious SD transmission peaks for FePc-FePc MDs in the PC, therefore, just the PDOS peaks at or around the transmission peak make a contribution to the transmission, and the FePc-FePc MD in the APC appears the same situation and is shown in Figure 4d. The transmission is mainly controlled by the PDOS of the $\mathrm{p}$ orbital. 

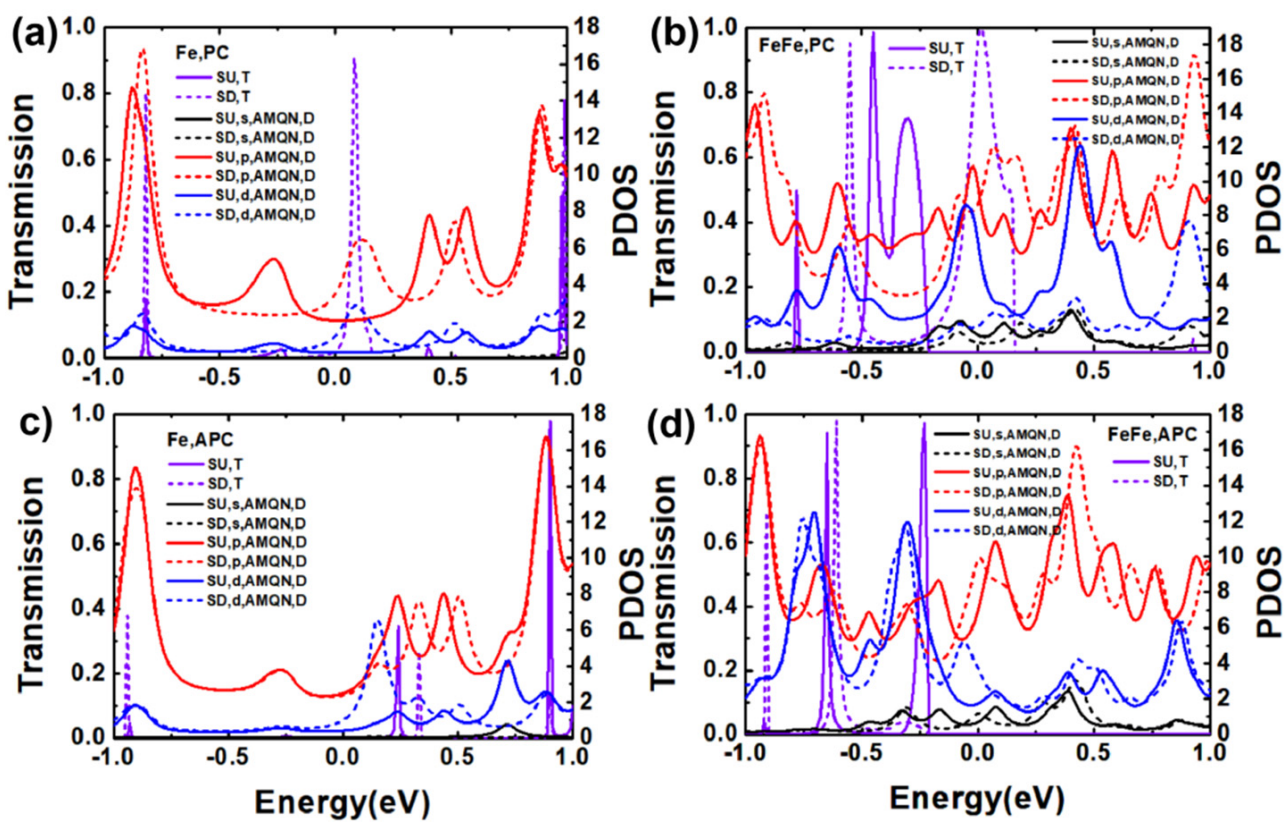

Figure 4. The spin-dependent transmission (T) and projected density of state (D) for s-, p-, d-orbital of the scattering region of the FePc and FePc-FePc MD in the PC $(\mathbf{a}, \mathbf{b})$ and APC (c,d) at zero bias.

Figure 5 shows the real-space scattering states of FePc and FePc-FePc molecular devices in the PC and APC at zero bias. Figure 5a demonstrates that the SD scattering of incoming state of lead L (SSLL) and lead R (SSLR) of the FePc MD in the PC are larger than the $\mathrm{SD}$ one, which corresponds to the fact that the SD G is greater than the SU G of the FePc MD in the PC. The SU SSLL and SSLR of the FePc MD in the PC shown in Figure 5a is smaller than the ones in the APC shown in Figure 5b, the SD SSLL and SSLR of the FePc $\mathrm{MD}$ in the PC is larger than the ones in the APC, which corresponds the SU G of the FePc MD in the PC being lower than the one in the APC and the SD G of the FePc MD in the $\mathrm{PC}$ is greater than the one in the APC. Figure $5 \mathrm{c}$, d shows a similar situation for FePc-FePc MDs in the PC and APC. Meanwhile, the SD SSLL and SSLR of FePc-FePc MDs in the PC and APC are greater than the ones of FePc in the PC and APC, which also explains why the $\mathrm{G}$ of the FePc-FePc MD is larger than the one of the FePc MD in the PC and APC.

Figure $6 \mathrm{a}, \mathrm{b}$ demonstrates the spin-dependent Seebeck coefficient $S_{\sigma}$ in the left column, the spin-dependent charge Seebeck coefficient $S_{C}$ and the spin Seebeck coefficient $S_{S}$ in the right column of $\mathrm{CuPc}, \mathrm{CoPc}, \mathrm{FePc} \mathrm{MD}$ in the $\mathrm{PC}$ and $\mathrm{APC}$ versus the temperature at zero bias. Generally, shown in Figure 6a, for the $\mathrm{S}_{\downarrow}$ of $\mathrm{CuPc}$ and $\mathrm{CoPc}$ in the PC, the latter is quite small, $\mathrm{S}_{\downarrow} \approx 0$. The $\mathrm{S}_{\uparrow}$ of CuPc, CoPc, FePc MD in the PC first decreases, then increases and then decreases with $\mathrm{T}$. $\mathrm{S}_{\mathrm{C}}=0$ at $\mathrm{T} \approx 307$ and $482 \mathrm{~K}$ for $\mathrm{CuPc} \mathrm{MD}$ and $\mathrm{S}_{\mathrm{C}}=0$ at $\mathrm{T} \approx 218 \mathrm{~K}$ for FePc MD in the $\mathrm{PC}$, a pure spin current produced by the temperature gradient. For CoPc MD in the $\mathrm{PC}, \mathrm{S}_{\mathrm{C}} \approx \mathrm{S}_{\mathrm{S}}$ duo to $\mathrm{S}_{\downarrow}$ is close to zero and described by the formula of $S_{C}$ and $S_{S}$. As shown in Figure 6a, at a low temperature, $T, S_{\sigma}$ is linear in $T$ [32] and there are large and negative $S_{\uparrow}$ and $S_{\downarrow}$ around $T=160 \mathrm{~K}$ for CuPc MD, large $S_{\uparrow}$ and $S_{\downarrow}$ around $\mathrm{T}=211$ and $261 \mathrm{~K}$ for CoPc MD, and a large $S_{\downarrow}$ around $\mathrm{T}=207 \mathrm{~K}$ and a large and negative $S_{\uparrow}$ around $207 \mathrm{~K}$ for FePc MD in the APC. There is a pure spin current with $\mathrm{S}_{\mathrm{C}}=0$ at $\mathrm{T} \approx 100 \mathrm{~K}$ for CuPc MD, at $\mathrm{T} \approx 143 \mathrm{~K}$ for CoPc MD, at $\mathrm{T} \approx 99 \mathrm{~K}$ for FePc MD and a large and negative $S_{C}$ and a small and negative $S_{S}$ at $T=161 \mathrm{~K}$ for CuPc MD, a large $S_{C}$ and a small $\mathrm{S}_{\mathrm{S}}$ at $\mathrm{T}=500 \mathrm{~K}$ for CoPc MD, a large and negative $\mathrm{S}_{\mathrm{S}}$ and a small and negative $\mathrm{S}_{\mathrm{C}}$ at $\mathrm{T}=206 \mathrm{~K}$ for FePc MD in the APC. 


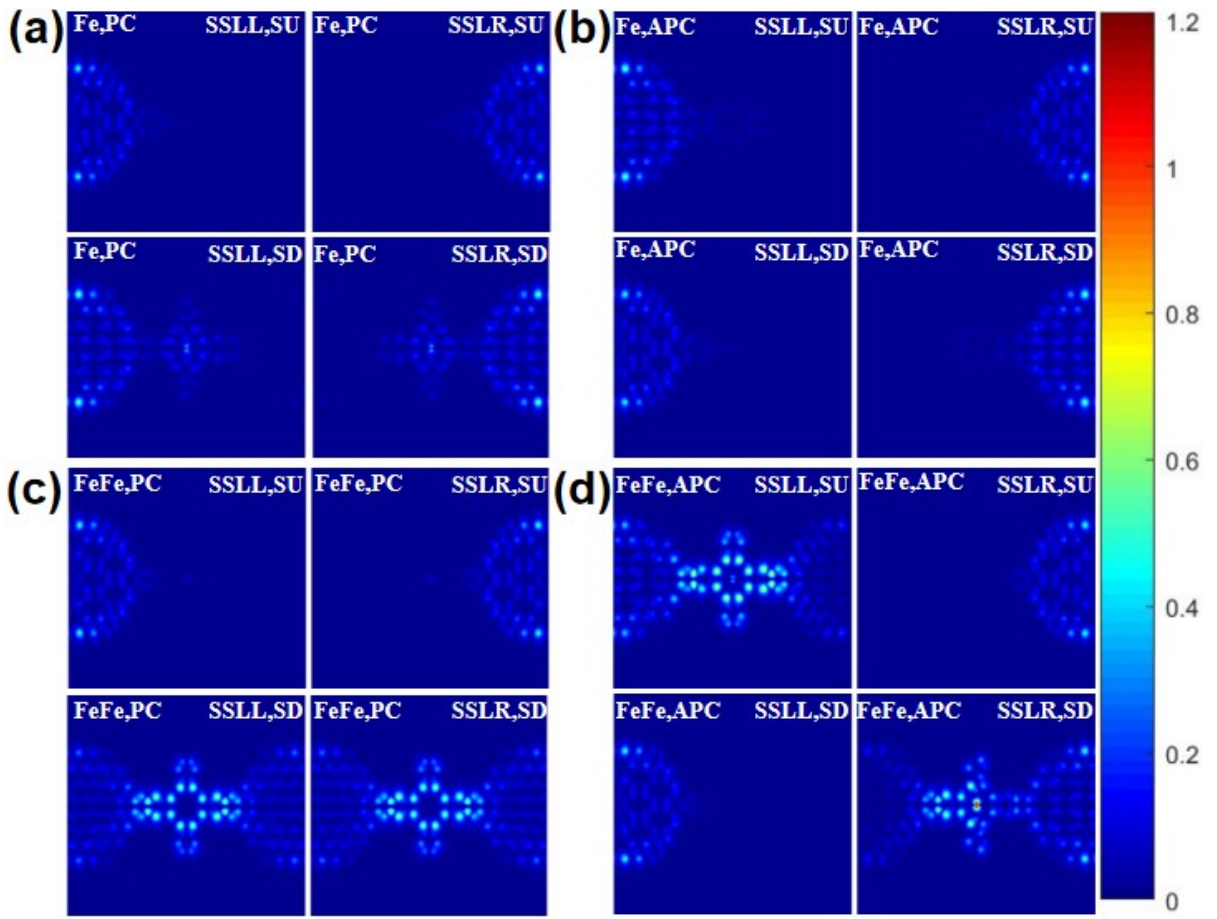

Figure 5. The scattering states of the FePc in the PC (a) and APC (b), and FePc-FePc MDs in the PC (c) and APC (d) at zero bias.

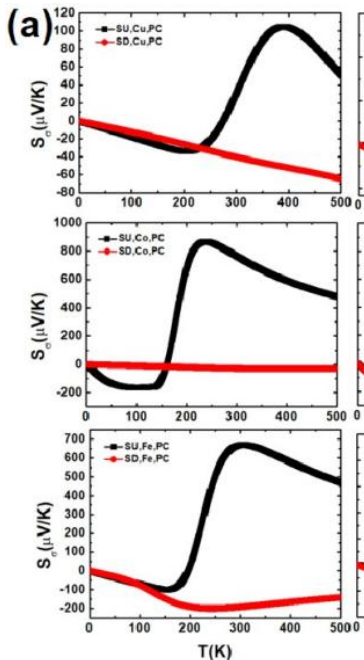

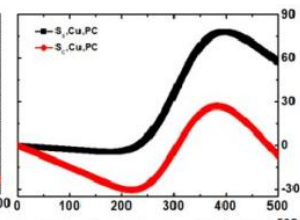
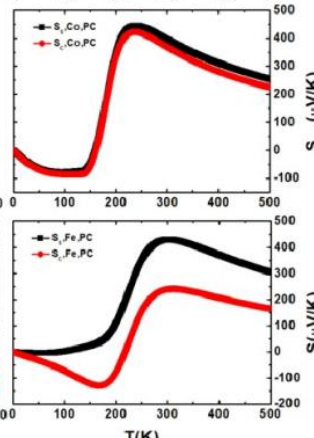

$T(K)$

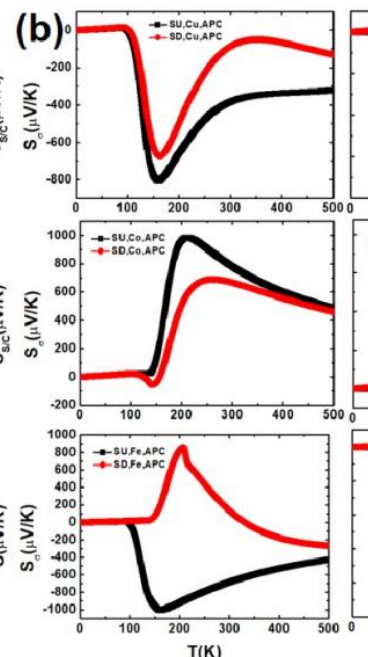

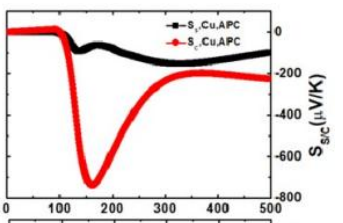

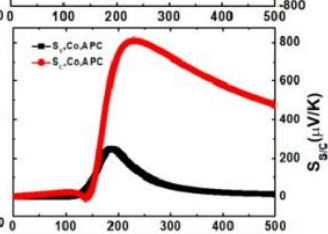

Figure 6. The spin-dependent Seebeck coefficient $S_{\sigma}$, charge Seebeck coefficient $S_{C}$ and the spin Seebeck coefficient $\mathrm{S}_{\mathrm{S}}$ of $\mathrm{CuPc}, \mathrm{CoPc}, \mathrm{FePc}$ MD in the PC (a) and APC (b) at zero bias.

Figure $7 \mathrm{a}, \mathrm{b}$ shows that the $\mathrm{S}_{\sigma}$ in the left column, the $\mathrm{S}_{\mathrm{C}}$ and $\mathrm{S}_{\mathrm{S}}$ in the right column of $\mathrm{CuPc}-\mathrm{CuPc}$, CoPc-CoPc, FePc-FePc, CuPc-CoPc, CuPc-FePc, CoPc-FePc MD in the PC and APC versus the temperature at zero bias. The $S_{\downarrow}$ of the bilayer MPc MD is close to zero and $S_{C} \approx S_{S}$ in the PC in Figure 7a,b. Interestingly, the $S_{S}$ is close to zero in most regions of the temperature range. There is a tendency to produce a pure charge current with the increasing temperature and $\mathrm{S}_{\downarrow} \approx \mathrm{S}_{\uparrow}$ for $\mathrm{CoPc}-\mathrm{CoPc}, \mathrm{CuPc}-\mathrm{CoPc}, \mathrm{CuPc}-\mathrm{FePc}, \mathrm{CoPc}-\mathrm{FePc} \mathrm{MDs}$ in the APC, as shown in Figure 7c,d. 

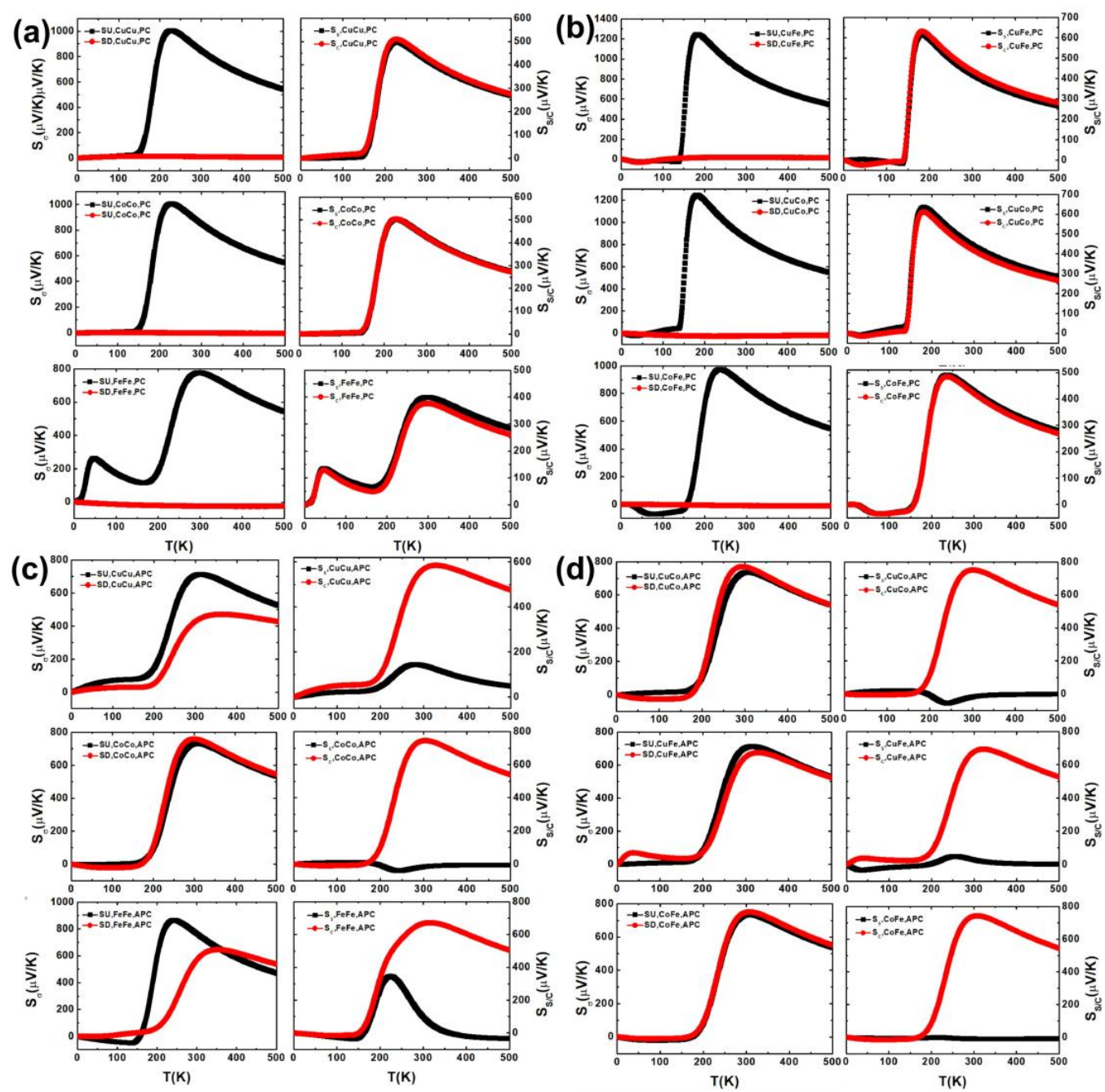

Figure 7. The spin-dependent Seebeck coefficient $S_{\sigma}$, charge Seebeck coefficient $S_{C}$ and the spin Seebeck coefficient $\mathrm{S}_{\mathrm{S}}$ of $\mathrm{CuPc}-\mathrm{CuPc}$, CoPc-CoPc, FePc-FePc, CuPc-CoPc, CuPc-FePc, CoPc-FePc MDs in the PC $(\mathbf{a}, \mathbf{b})$ and APC $(\mathbf{c}, \mathbf{d})$ at zero bias.

Therefore, we can obtain an ultrahigh spin filter efficiency exceeding $99.99995 \%$ and ultra-large total conductance of 0.49996G0 for FePc-CoPc molecular device in the PC and a nearly pure charge current at the high temperature in the APC as well as a giant MR ratio exceeding $9.87 \times 10^{6 \%}$ at zero bias. There are pure spin currents for $\mathrm{CuPc}$ and $\mathrm{FePc}$ molecular devices in the PC, and an almost pure spin current for FePc molecular device in the APC at some temperatures. Meanwhile, there is a high SFE of about $99.99585 \%$ in the PC and a reserved SFE of about $-19.533 \%$ in the APC as well as a maximum MR ratio of about $3.69 \times 10^{8} \%$ for monolayer FePc molecular device. In addition, the spin-dependent charge Seebeck coefficient $\mathrm{S}_{\mathrm{C}}$ and the spin Seebeck coefficient $\mathrm{S}_{\mathrm{S}}$ are almost equal for CoPc, $\mathrm{CuPc}-\mathrm{CuPc}$, CoPc-CoPc, FePc-FePc, CuPc-CoPc, CuPc-FePc, CoPc-FePc molecular devices in the PC duo to the spin-down Seebeck coefficient is close to zero and there are usually large charge Seebeck coefficients at high temperatures for the above molecular devices in the APC.

\section{Conclusions}

In conclusion, we investigated the spin-dependent conductance, spin filter efficiency, giant magnetoresistance ratio, Seebeck coefficient, charge Seebeck coefficient and spin Seebeck coefficient by analyzing the projected density of state, transmission spectrum and scattering state of monolayer and bilayer MPc $(\mathrm{M}=\mathrm{Fe}, \mathrm{Co}, \mathrm{Cu})$ molecular devices in the parallel and anti-parallel spin configurations by employing LDA, LDA + U and 
nonequilibrium Green's function approaches. These methods have their benefits over other approaches for nanomaterials with transition metals, Fe in particular. The results show that the spin filter efficiency in the parallel spin configuration, spin-down and total conductance of the bilayer MPc molecular devices were superior to the monolayer MPc molecular device. There are large pure spin currents in the $\mathrm{CuPc}$ and FePc molecular devices in the parallel spin configuration at some temperatures. Meanwhile, there is a high SFE of about $99.99585 \%$ in the parallel spin configuration and a reserved SFE of about $-19.533 \%$ in the anti-parallel spin configuration and a maximum MR ratio of about $3.69 \times 10^{8 \%}$ for monolayer FePc molecular devices. These transport phenomena could be well understood by analyzing the transmission spectra, projected density of state and scattering states. The calculated transmission spectra and the real-space scattering states of the modeled mono- and bilayers can be used for future experimental photoemission spectroscopy and other studies. These theoretical calculations predict new monolayer and bilayer phthalocyanine-based molecular devices with high MR and Seebeck coefficients. This makes the results motivating for further experimental studies of phthalocyanine and transition metal layered structures. The obtained large spin-filter efficiency of the parallel spin configuration can be utilized in various molecular spintronic devices.

Author Contributions: Conceptualization, J.L. and Z.W.; methodology, J.L.; software, J.L., K.L. and Z.W.; validation, J.L.; formal analysis, J.L.; investigation, J.L.; resources, J.L.; data curation, J.L.; writing—original draft preparation, J.L.; writing—review and editing, J.L., K.L., H.C., B.S. and Z.W.; visualization, J.L.; supervision, K.L.; project administration, H.C., B.S. and Z.W.; funding acquisition, H.C., B.S. and Z.W. All authors have read and agreed to the published version of the manuscript.

Funding: This work was supported by the National Key Research and Development Program of China under Grant No. 2016YFA0201903 and 2016YFA0202304, General Program of National Natural Science Foundation of China under Grant No. 61674168 and 61504165, Research and Development Project in Key Fields of Guangdong Province under Grant No. 2020 B010171001.

Institutional Review Board Statement: Not applicable.

Informed Consent Statement: Not applicable.

Data Availability Statement: The data is available on reasonable request from the authors. The data are not publicly available due to ethical.

Conflicts of Interest: The authors declare no conflict of interest.

\section{References}

1. Wolf, S.A.; Awschalom, D.D.; Buhrman, R.A.; Daughton, J.A.; von Molnár, S.; Roukes, M.L.; Chtchelkanova, A.Y.; Treger, D.M. spintronics: A spin-based electronics vision for the future. Science 2001, 294, 1488-1495. [CrossRef]

2. Lakshmi, S.; Roche, S.; Cuniberti, G. Spin-valve effect in zigzag graphene nanoribbons by defect engineering. Phys. Rev. B 2009, 80, 193404. [CrossRef]

3. Rocha, A.R.; Garcia-Suárez, V.M.; Bailey, S.W.; Lambert, C.J.; Ferrer, J.; Sanvito, S. Towards molecular spintronics. Nat. Mater. 2005, 4, 335. [CrossRef]

4. Atodiresei, N.; Brede, J.; Lazic, P.; Caciuc, V.; Hoffmann, G.; Wiesendanger, R.; Blugel, S. Design of the local spin polarization at the organic-ferromagnetic interface. Phys. Rev. Lett. 2010, 105, 066601. [CrossRef]

5. Wan, H.Q.; Zhou, B.H.; Chen, X.W.; Sun, C.Q.; Zhou, G.H. Switching, Dual Spin-Filtering Effects, and Negative Differential Resistance in a Carbon-Based Molecular Device. J. Phys. Chem. C 2012, 116, 2570. [CrossRef]

6. Shen, X.; Sun, L.L.; Yi, Z.L.; Benassi, E.; Zhang, R.X.; Shen, Z.Y.; Sanvito, S.; Hou, S.M. Spin transport properties of 3d transition metal (II) phthalocyanines in contact with single-walled carbon nanotube electrodes. Phys. Chem. Chem. Phys. 2010, $12,10805$. [CrossRef]

7. Zhou, Y.H.; Zeng, J.; Tang, L.M.; Chen, K.Q.; Hu, W.P. Giant magnetoresistance effect and spin filters in phthalocyanine-based molecular devices. Org. Electron. 2013, 14, 2940-2947. [CrossRef]

8. Liu, J.H.; Luo, K.; Huang, K.L.; Sun, B.; Zhang, S.L.; Wu, Z.H. Tunable conductance and spin filtering in twisted bilayer copper phthalocyanine molecular devices. Nanoscale Adv. 2021, 3, 3497-3501. [CrossRef]

9. Schmaus, S.; Bagrets, A.; Nahas, Y.; Yamada, T.K.; Bork, A.; Bowen, M.; Beaurepaire, E.; Evers, F.; Wulfhekel, W. Giant magnetoresistance through a single molecule. Nat. Nanotechnol. 2011, 6, 185-189. [CrossRef]

10. Tao, L.L.; Wang, J. Giant magnetoresistance and perfect spin filter effects in manganese phthalocyanine based molecular junctions. Nanoscale 2017, 9, 12684-12689. [CrossRef] 
11. Sun, X.W.; Zhao, P. Large dual spin-rectifying and high-efficiency dual spin-filtering in cyclooligomeric Mn-phthalocyanine dimer molecular junction. Chem. Phys. Lett. 2019, 724, 73-79. [CrossRef]

12. Zhao, P.; Chen, G. Spin-polarized and thermospin-polarized transport properties of phthalocyanine dimer based molecular junction with different transition metal atoms. J. Chem. Phys. 2018, 149, 134305. [CrossRef]

13. Xiao, Y.; Liu, L.; Ma, Z.H.; Meng, B.; Qin, S.J.; Pan, G.B. High-Performance Self-Powered Ultraviolet Photodetector Based on Nano-Porous GaN and CoPc p-n Vertical Heterojunction. Nanomaterials 2019, 9, 1198. [CrossRef]

14. Hohenberg, P.; Kohn, W. Inhomogeneous Electron Gas. Phys. Rev. 1964, 136, 864-871. [CrossRef]

15. Kohn, W.; Sham, L.J. Self-consistent equations including exchange and correlation effects. Phys. Rev. 1965, 140, 1133-1138. [CrossRef]

16. NanoDCAL. Available online: https://www.nanoacademic.com/product-page/nanodcal (accessed on 16 September 2021).

17. Kirner, J.F.; Dow, W.; Scheidt, W.R. Molecular stereochemistry of two intermediate-spin complexes. Iron(II) phthalocyanine and manganese(II) phthalocyanine. Inorg. Chem. 1976, 15, 1685-1690. [CrossRef]

18. Mason, R.; Williams, G.A.; Fielding, P.E. Structural chemistry of phthalocyaninato-cobalt(II) and -manganese(II). J. Chem. Soc. Dalton Trans. 1979, 676. [CrossRef]

19. Mastryukov, V.; Ruan, C.Y.; Fink, M.; Wang, Z.; Pachter, R. The molecular structure of copper- and nickel-phthalocyanine as determined by gas-phase electron diffraction and ab initio/DFT computations. J. Mol. Struct. 2000, 556, 225-237. [CrossRef]

20. Qi, P.; Javey, A.; Rolandi, M.; Wang, Q.; Dai, H. Miniature organic transistors with carbon nanotubes as quasi-one dimensional electrodes. J. Am. Chem. Soc. 2004, 126, 11774-11775. [CrossRef]

21. Cho, Y.; Kim, W.Y.; Kim, K.S. Effect of electrodes on electronic transport of molecular electronic devices. J. Phys. Chem. A 2009, 113, 4100. [CrossRef]

22. Tsuyuki, H.; Shiibashi, T.; Sakamoto, S.; Tomiya, M. Effects of substitutional doping in electronic transport properties of carbon nanotubes. Int. J. Mod. Phys. B 2013, 27, 1350157. [CrossRef]

23. Cui, L.L.; Yang, B.C.; Li, X.M.; He, J.; Long, M.Q. Electronic transport properties of transition metal (Cu, Fe) phthalocyanines connecting to V-shaped zigzag graphene nanoribbons. Int. J. Mod. Phys. B 2014, 28, 1450019. [CrossRef]

24. Liu, W.; Jackson, B.L.; Zhu, J.; Miao, C.Q.; Chung, C.H.; Park, Y.J.; Sun, K.; Woo, J.; Xie, Y.H. Large scale pattern graphene electrode for high performance in transparent organic single crystal field-effect transistors. ACS Nano 2010, 4, 3927-3932. [CrossRef]

25. Lee, W.H.; Park, J.; Sim, S.H.; Jo, S.B.; Cho, K. Transparent flexible organic transistors based on monolayer graphene electrodes on plastic. Adv. Mater. 2011, 23, 1752-1756. [CrossRef] [PubMed]

26. Taylor, J.; Guo, H.; Wang, J. Ab initio modeling of quantum transport properties of molecular electronic devices. Phys. Rev. B 2001, 63, 245407. [CrossRef]

27. Cococcioni, M.; de Gironcoli, S. Linear response approach to the calculation of the effective interaction parameters in the LDA + U method. Phys. Rev. B 2005, 71, 035105. [CrossRef]

28. Wang, Y.; Li, X.G.; Zheng, X.; Yang, J.L. Manipulation of spin and magnetic anisotropy in bilayer magnetic molecular junctions Phys. Chem. Chem. Phys. 2018, 20, 26396. [CrossRef] [PubMed]

29. Tao, L.L.; Liang, S.H.; Liu, D.P.; Han, X.F. Large magnetoresistance of paracyclophane-based molecular tunnel junctions: A first principles study. J. Appl. Phys. 2013, 114, 213906. [CrossRef]

30. Zhai, M.X.; Wang, X.F.; Vasilopoulos, P.; Liu, Y.S.; Dong, Y.J.; Zhou, L.P.; Jiang, Y.J.; You, W.L. Giant magnetoresistance and spin Seebeck coefficient in zigzag $\alpha$-graphyne nanoribbons. Nanoscale 2014, 6, 11121-11129. [CrossRef]

31. Zhao, C.C.; Tan, S.H.; Peng, X.F.; Wang, X.J.; Long, M.Q. Spin filter type transformation in Sn-phthalocyanine. Org. Electron. 2017, 43, 47-54. [CrossRef]

32. Yang, X.F.; Liu, Y.S. Pure spin current in a double quantum dot device generated by thermal bias. J. Appl. Phys. 2013, 113, 164310. [CrossRef] 